\title{
Características Anatómicas-Funcionales que Orientan la Posición del Cóndilo en la Fosa Mandibular en una Relación Céntrica. Una Descripción Narrativa
}

\author{
Anatomical-Functional Characteristics that Guide the Condylar Position \\ in Mandibular Fossa a Centric Relation. Narrative Description
}

Constanza Farfán ${ }^{1,2}$; Bryan Quidel ${ }^{3}$ \& Ramón Fuentes $^{1}$

FARFÁN, C.; QUIDEL, B. \& FUENTES, R. Características anatómicas-funcionales que orientan la posición del cóndilo en la fosa mandibular en una relación céntrica. Una descripción narrativa. Int. J. Morphol., 38(5):1281-1287, 2020.

RESUMEN: La Relación Céntrica (RC) es un concepto que busca definir la posición ideal del cóndilo de la mandíbula en la fosa mandibular, siendo esta posición muy discutida en la literatura y ha ido cambiado con los avances en conocimiento y tecnología. Tomándose en la actualidad en consideración elementos anatómicos esenciales que son utilizados como guía para el clínico. La importancia de poder reconocer esta posición radica en la correcta ejecución tanto de procedimientos diagnósticos como de tratamiento en diversas áreas de la odontología. Existen elementos anatómicos de la articulación temporomandibular (ATM) que nos ayudarían a comprender mejor el concepto de RC, entre estos destacan que la condensación ósea de las superficies articulares funcionales y su recubrimiento por fibrocartílago; la porción media del disco articular, desprovista de vasos y nervios; el techo de la fosa mandibular que puede llegar a ser extremadamente delgado por lo cual no se consideraría un área para soportar carga; la prolongación posterior del disco articular (DA) que es altamente vascularizada e inervada. El DA se encuentra íntimamente relacionado con estructuras como el ligamento temporomandibular o lateral y la cabeza superior del músculo pterigoideo lateral que permitirían junto a los elementos anteriormente mencionados el adecuado funcionamiento de la ATM. El correcto aprendizaje y utilización de estos conceptos contribuirá a lograr un mejor ejercicio del desarrollo profesional de estudiantes y profesionales del área de la odontología.

PALABRAS CLAVE: Relación Céntrica; Anatomía funcional; Articulación Temporomandibular; Fisiología oral.

\section{INTRODUCCIÓN}

La relación céntrica $(\mathrm{RC})$ es un concepto clínico en odontología, que busca definir la posición ideal del cóndilo de la mandíbula (CM) en la fosa mandibular del hueso temporal (Orozco Varo et al., 2008a,b). Considerada una posición de referencia mandibular esencial para la evaluación y tratamientos en diferentes especialidades odontológicas, como rehabilitación oral y ortodoncia (Rinchuse \& Kandasamy, 2006; Caravadossi et al., 2012), este concepto ha sido tema de discusión, y ha ido cambiando en el tiempo.

Uno de los primeros autores en definirla fue McCollum (1938), quien indicaba que durante la RC los cóndilos estaban localizados en una posición posterior en la fosa glenoidea. Diferentes autores apoyaron esta teoría, como Lucia (1960), quien consideraba que cuando los centros de rotación condilar estaban en el eje terminal de bisagra había
RC. Con el transcurso de los años, se demostró que la porción posterior de la cavidad articular está muy vascularizada e inervada (Okesson, 2003), por lo que esta teoría fue cuestionada por diferentes autores (Dawson, 1974; Orozco Varo et al., 2008a,b); uno de los primeros en plantear y definir como válida la posición de los cóndilos más superior, contra la eminencia, independiente de la posición dentaria, fue Peter Dawson (1974), quien posteriormente amplio su definición agregando el concepto de medial (Dawson, 1985). Existe además, una definición en el glosario de términos de prostodoncia (GTP), que desde que se publicó el año 1987 (Glossary of Prosthodontic Terms, 1987) ha tenido varias ediciones posteriores, la más actual es del año 2017 y define a la RC como una relación maxilomandibular, independiente del contacto dental, en la que los cóndilos se articulan en la posición supero-anterior contra las pendientes posterio-

\footnotetext{
${ }^{1}$ Department of Integral Adults Dentistry, Research Centre for Dental Sciences (CICO), Universidad de La Frontera, Temuco, Chile.

${ }^{2}$ Universidad Adventista de Chile, Chillán, Chile.

${ }^{3}$ Undergraduate student, Dental School, Universidad de La Frontera, Temuco, Chile.
} 
res de las eminencias articulares; posición en la que la mandíbula está restringida a un movimiento puramente rotatorio; es una posición clínica de referencia útil y repetible (The Glossary of Prosthodontic Terms: Ninth Edition, 2017).

Diferentes autores han establecido requisitos para adaptar la posición de RC, afirmando que es una posición predecible y estable. De esta forma Dawson establece cuatro requisitos para que la mandíbula se encuentre en $\mathrm{RC}: 1$ ) El disco articular (DA) debe estar alineado sobre ambos cóndilos. 2) El complejo articular está en el punto más alto posible contra la vertiente posterior de la eminencia. 3) El polo medial de cada complejo articular está abrazado por hueso. 4) La cabeza inferior del músculo pterigoideo lateral debe estar en reposo (Dawson, 1995). También el GTP indica que es una posición fisiológica maxilomandibular cuando: 1) Ambos cóndilos articulan con la porción más delgada y avascular de sus respectivos discos. 2) Esta posición es independiente de los contactos dentarios (Glossary of Prosthodontic Terms). Okesson concuerda con estos autores, ampliando el término de RC, destacando la importancia del DA y la neuromusculatura como elementos esenciales para la definición del concepto de forma fisiológica. Informando que el DA debe estar interpuesto adecuadamente y con una contracción intensa de los músculos elevadores, logrando una posición estable de la mandíbula (Okesson, 2003).

Junto con conocer la definición del concepto y la posición ideal del cóndilo de la mandíbula dentro de la fosa mandibular, resulta esencial estudiar los elementos anatómicos que orientan al clínico para lograr una adecuada RC, de acuerdo a esto el objetivo de este artículo es describir las características anatómicas-funcionales que orientan la posición del cóndilo en la fosa mandibular en una relación céntrica.

\section{RELACIÓN CÉNTRICA}

Uno de los términos más controversiales en odontología general y en la prostodoncia en particular, es la RC y no sólo en lo referente a su definición sino también al procedimiento para registrarla, labor en la que han trabajado numerosos autores con el objetivo de encontrar la manera más fiable de reproducir dicha posición (Orozco Varo et al., 2008a,b; Caravadossi et al.).

Características anatómicas-funcionales. La importancia de establecer la RC, radica principalmente en la planificación de tratamientos odontológicos, es la base para programar e iniciar los tratamientos de rehabilitación, ayuda a los clínicos a evitar complicaciones de tipo muscular o articu- lar durante el procedimiento propuesto. Para lograr esta posición es necesaria la ayuda de los músculos mandibulares, que colaboran estabilizando la mandíbula en la posición de $\mathrm{RC}$ original del sujeto, que, dependiendo de las características del paciente, puede verse modificada, por lo tanto, se vuelve necesaria lograr identificar la nueva RC del paciente. La predictibilidad de todos los tipos de tratamientos oclusales (ajuste oclusal, férulas oclusales, coronas, inlay, onlay y overlay) se encuentran clínicamente relacionadas con la capacidad del odontólogo para determinar de forma precisa la RC, ya que esta es una guía para el montaje de estudio de los modelos del paciente en el articulador. La RC tiene como finalidad otorgar una posición musculo esquelética estable proporcionando un equilibrio posicional a la articulación (Okesson, 2013), además de ser un método de referencia para establecer una relación mandibular horizontal y vertical equilibrada (Kandasamy et al., 2018).

Las técnicas utilizadas en práctica clínica para determinar la RC, son variadas y suelen ser dificultosas al principio, sin embargo, son necesarias para llevar a cabo un tratamiento basado en la oclusión funcional (Caravadossi et al.), se pueden agrupar básicamente en 3 categorías, aquellas inducidas por "Manipulación Mandibular", donde encontramos técnicas como la guía del mentón o "Chin Point" y Técnica Bimanual de Dawson. "Las electroinducidas" compuesta por la Técnica Miocéntrica, y finalmente las "Autoinducidas por desprogramación neuromuscular" donde destaca la técnica de "Power Centric" o Céntrica de fuerza, Jig Incisal o desprogramador anterior, plano interoclusal y las "Laminillas de Long” (Manns \& Biotti, 2006).

A continuación, presentaremos las características anatómicas que se deben considerar al momento de establecer la RC:

Condensación ósea de superficies funcionales. La vertiente posterior del tubérculo articular alcanza un espesor $0,50 \mathrm{~mm}$ y en la carilla articular del cóndilo de la mandíbula (CM) un espesor de 2,00 mm (Fig. 1) (Gómez \& Campos, 2009), esta área del tubérculo articular, corresponde a un área de bastante grosor diseñada para soportar una fuerza fisiológica (Okesson, 2013).

El crecimiento del cóndilo de la mandíbula requiere un mecanismo de crecimiento endocondral, este crece en dirección a la articulación (Fig. 1), que está sometida a una presión directa, este crecimiento sólo ocurre a nivel de las superficies articulares, ya que este es el lugar donde se ejerce una presión sobre la tolerancia de la membrana del tejido blando del hueso, un crecimiento de tipo intramembranoso a este nivel no sería capaz de soportar las presiones que se ejercen en este lugar (Aguila \& Enlow, 1993). 


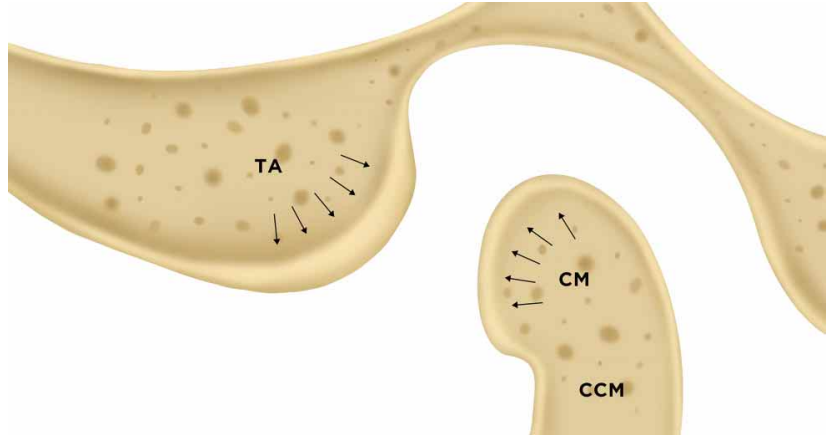

Fig 1. Corte sagital ATM Izquierda. Donde se representa la dirección de crecimiento de las superficies articulares funcionales $(* * * \rightarrow)$ del Tubérculo Articular (TA) y Cóndilo de la mandíbula (CM) y en color amarillo pálido es posible diferenciar el espesor de las superficies articulares (TA y CM).

Recubrimiento superficies funcionales. Las superficies articulares de la ATM, tienen diferentes recubiertas dependiendo si son funcionales o no, las funcionales están recubiertas de fibrocartílago (Langton \& Eggleton, 1992) y las no articulares por tejido fibroso (Velayos \& Santana, 2001) (Fig. 2). Ambas superficies articulares, tanto mandibular como temporal, están constituidas por 4 capas o zonas distintas, entre ellas encontramos la Zona Articular, Proliferativa, Fibrocartilaginosa y Calcificada (Okesson, 2013). Estas superficies articulares están cubiertas por un periostio modificado con una zona subyacente de fibrocartílago, destinada a soportar carga, donde la superficie articular del cóndilo de la mandíbula correspondería a la capa fibrosa del periostio o zona articular y la zona proliferativa subyacente la capa del "cambium", esta última estaría encargada de suministrar células tanto para la capa fibrosa del periostio como para el fibrocartílago subyacente (Stocum \& Roberts, 2018).

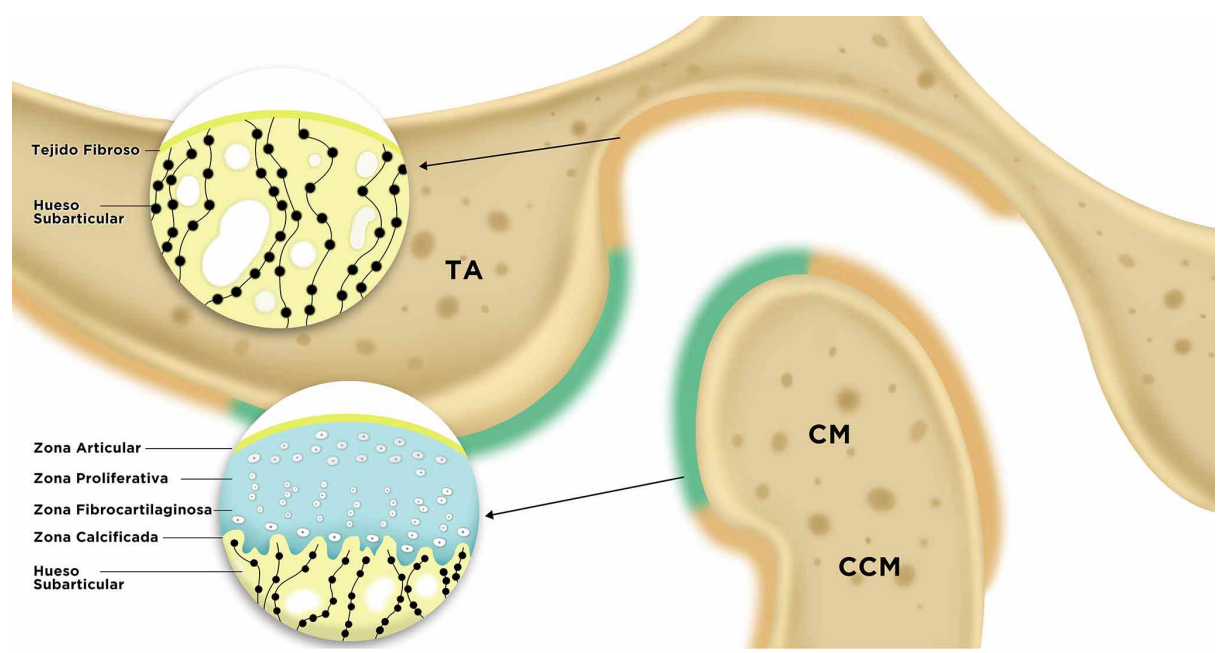

Fig. 2. Corte sagital ATM Izquierda. Caracterización del recubrimiento de superficies articulares funcionales y no funcionales de ATM.
Porción media disco articular. El disco articular está compuesto por fibrocartílago desprovisto de vasos sanguíneos y cuenta con solo una ligera inervación en la zona más periférica (Okesson 2013; Stocum \& Roberts, 2018), acompaña al cóndilo de la mandíbula en todos los movimientos mandibulares (Gomez, 2009). En un corte sagital, en sentido antero-posterior se pueden destinguir 3 zonas, una zona anterior (ZA) y posterior (ZP) conformada por tejido fibroso denso, una zona media o de trabajo (ZM) que es más delgada (Fig. 3), sin vasos ni nervios y la zona retrodiscal o bilaminar muy inervada y vascularizada (Matamala et al., 2006). La composición de las 3 zonas del DA es diferente. La zona intermedia destaca por ser la porción más viscosa, flexible y menos retitulada que las otras. La región lateral y media posee predominio de proteoglicanos como decorina y biglucano (Stankovic et al., 2013). La porción media o de trabajo además de ser la zona más delgada en espesor dentro de la morfología del DA, destaca por estar compuesta, al igual que el área articular mandibular y temporal, por una densa trama de fibras colágenas que tiene una disposición paralela a la superficie articular (Okesson, 2013; Gómez \& Campos).

Diversos autores han descrito la morfometría del DA en adultos. Matamala et al., el año 2006, informan un diámetro en sentido antero posterior de $14,46 \pm 1,43 \mathrm{~mm}$ y en sentido transversal de 20,08 $\pm 1,26 \mathrm{~mm}$. En cuanto al espesor del DA de adultos se ha estudiado que, en sentido sagital se diferencian 3 zonas, zona anterior (ZA), zona media (ZM), y zona posterior $(\mathrm{ZP})$ guarda una relación promedio de una proporción de 2:1:3, respectivamente (Isberg, 2003), con una ZA que tiene un espesor en rango de $1 \mathrm{~mm}$ a $2 \mathrm{~mm}$ (Gómez \& Campos), acompañado de una ZM de $0,2 \mathrm{~mm}$ a $1 \mathrm{~mm}$ y una zona ZP de 3 mm a 4 mm (Manns \& Díaz, 1983), Según lo reportado por Matalama et $a l$. , estas zonas tienen un espesor de $2,39 \pm 1,44 \mathrm{~mm}, 1,6 \pm$ 0,85 y $3,29 \pm 1,02 \mathrm{~mm}$ respectivamente. Las variabilidades de los espesores en los diferentes estudios se relacionan con el método utilizado, y características de la muestra como etnia, edad, sexo.

Los criterios que definen una posición normal del DA, independiente de las variaciones anatómicas, son un adecuado contacto de: la prominencia anterior del cóndilo de la mandíbula y el tubérculo articular, con la ZM del DA 
(Fig. 3), aquí, en la convergencia de estas superficies convexas, se genera la zona bicóncava del DA. Ocupando la ZP, el espacio entre el cóndilo de la mandíbula y el techo de la fosa mandibular, posteriormente, se observa la zona bilaminar (ZB).

Techo de la fosa mandibular. El techo de la fosa mandibular está compuesto por un hueso muy fino por lo cual no se consideraría un área desarrollada para soportar carga (Okesson, 2013), a pesar de contener el margen posterior del DA y cóndilo de la mandíbula (Fig. 4), actuaría como una estructura más bien pasiva, donde el cóndilo de la mandíbula y el tubérculo articular del hueso temporal serían la parte activa (Fuentes et al., 2015).

Formando parte del piso de la fosa craneal media, este techo presenta un adelgazamiento de la estructura ósea, casi translúcido que es considerada como una zona no funcional de la ATM, y en caso de que ocurra un traumatismo a este nivel debe considerarse evaluar su integridad, por la posibilidad de que se produzcan fisuras con salida de líquido cerebroespinal (Estrella, 2006). Por otra parte, si comparamos la densidad ósea que existe en el techo de la fosa mandibular esta es muy baja en comparación con la vertiente posterior del tubérculo articular y explicaría en parte porque no se habla del concepto de relación céntrica como la posición "más posterior, superior y medial" (Rubiano, 1993).

Prolongación posterior disco articular. Por detrás del DA encontramos la zona retrodiscal o bilaminar, compuesta de tejido conectivo laxo, muy inervada y vascularizada (Matamala et al.). Dentro de esta zona podemos reconocer dos láminas o fascículos (Fig. 5), una laminilla retrodiscal superior constituida tanto de fibras colágenas como por fibras elásticas que se orientan hacia la superficie temporal (Gómez \& Campos), y la laminilla retrodiscal inferior, compuesta exclusivamente por fibras colágenas que se unen desde el margen posteroinferior del DA, al límite posterior de la superficie articular del cóndilo de la mandíbula (Okesson, 2013), e incluso puede llegar a extenderse hasta el cuello de la mandíbula donde se unen al periostio del hueso (Gómez \& Campos). Esta disposición de los tejidos de esta zona permite que no ocurra un desplazamiento del cóndilo hacia "arriba y atrás", sin embargo, si un movimiento "abajo y atrás" como en algunas incoordinaciones o alteraciones del sistema muscular (Ramfjord \& Ash, 1972).

Cuando se aplica una fuerza o presión sobre la zona retrodiscal, se produce dolor y puede llegar generarse lesiones, capaces de producir diversas alteraciones (Okesson, 2013). Los signos y síntomas como la otalgia, vértigo y

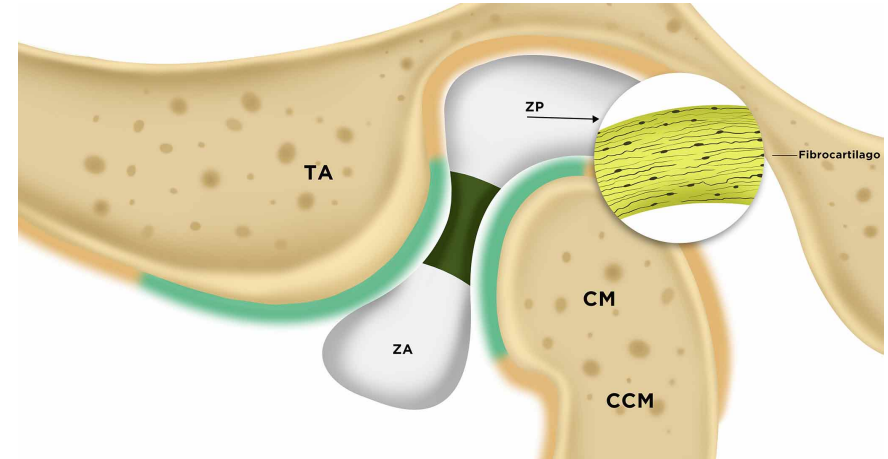

Fig. 3. Corte sagital de la ATM izquierda, se observa la relación de la Zona de trabajo o intermedia del Disco Articular (Verde) con las superficies del tubérculo articular (TA) y el cóndilo de la mandíbula (CM). Se observa el componente de la zona posterior (ZP) del disco articular y la superficie no articular con que se relaciona, además de la proporción: ZA: ZM: ZP (2:1:3).

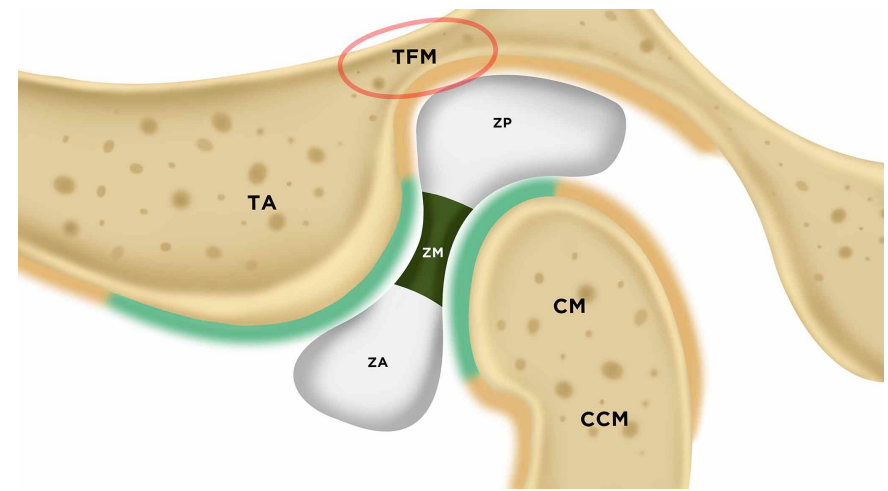

Fig. 4. Corte sagital ATM izquierda, donde se observa el espesor del hueso esponjoso del techo de techo de la fosa mandibular (TFM), su relación con la zona posterior (ZP) del disco articular.

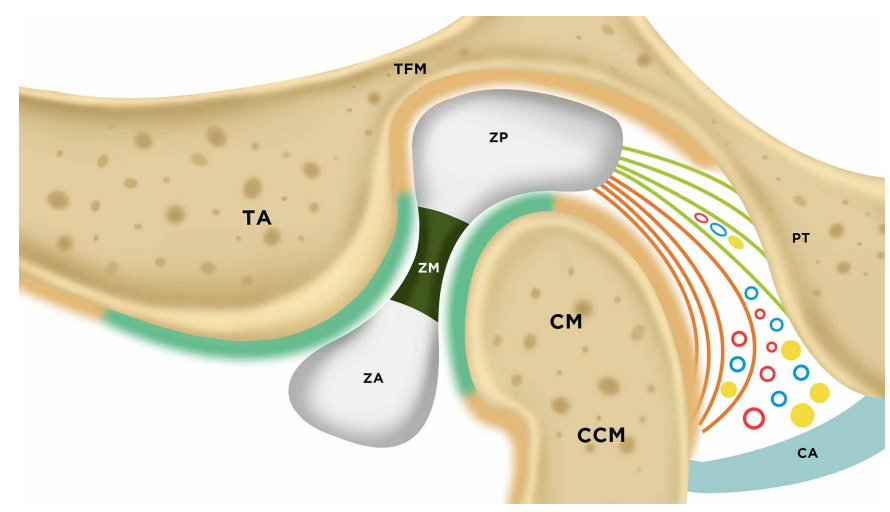

Fig. 5. Corte Sagital de la ATM Izquierda, donde se representa la prolongación posterior del Disco Articular: donde encontramos el paquete vasculo nervioso (o o o), la Zona Bilaminar, con su Laminilla Retrodiscal Superior ( - ); e Inferior ( - ), relacionándose con la cápsula articular (CA) y el cuello de la mandíbula (CCM). Es posible observar el cóndilo de la mandíbula (CM) y la relación de estas estructuras con el Pared Timpánica (PT). 


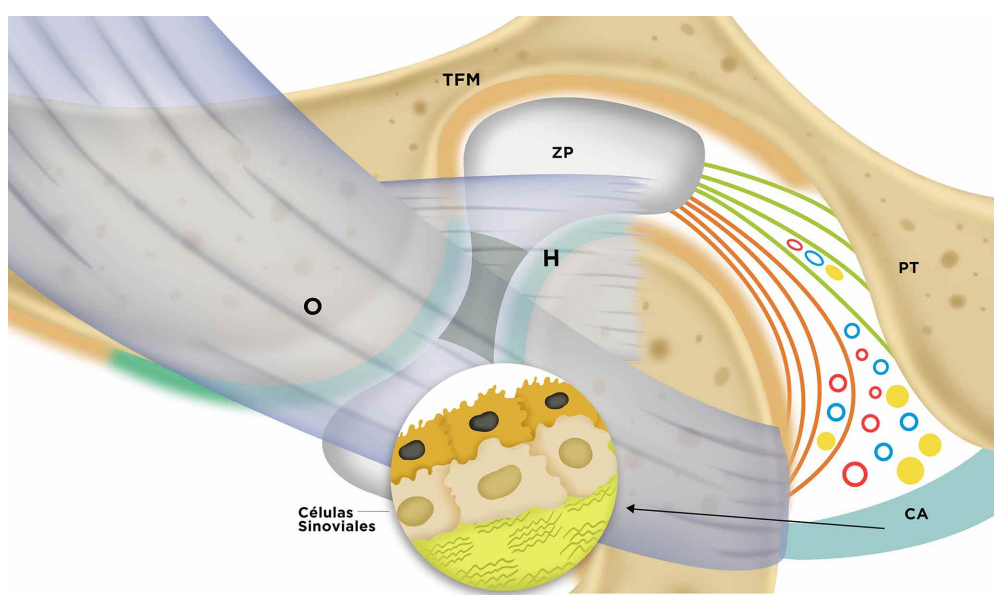

Fig. 6. Vista lateral de la ATM izquierda. Estructuras representadas: Ligamento Temporomandibular o Lateral, porción oblicua $(\mathrm{O})$ y horizontal $(\mathrm{H})$, y las células sinoviales de la CA.

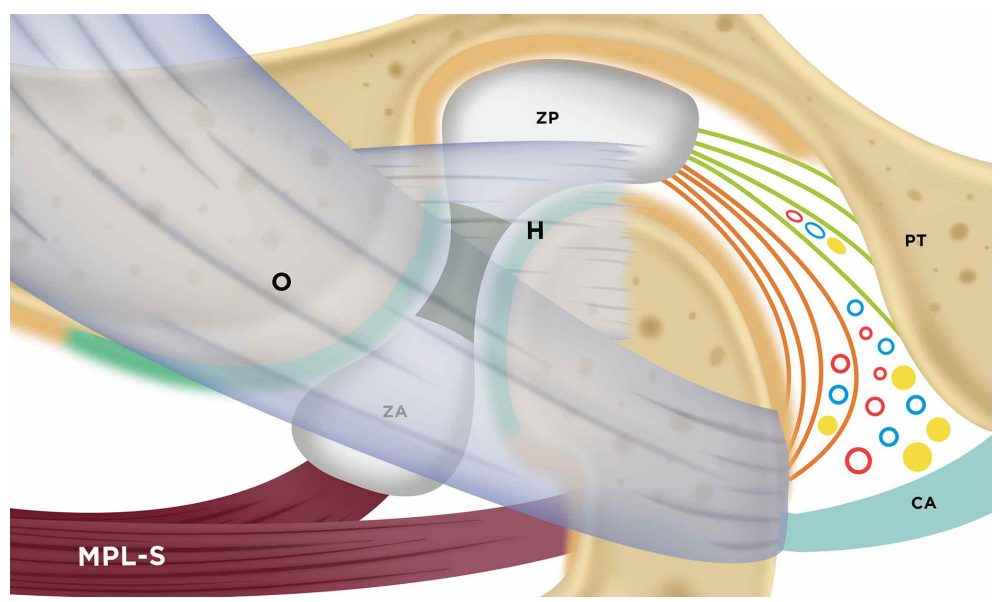

Fig. 7. Corte Sagital de la ATM izquierda, donde se puede observar la relación de la zona anterior (ZA) del disco articular y cuello de la mandíbula (CCM) con la cabeza superior del músculo pterigoideo lateral (MPL-S).

tinnitus, podrían ser resultado de alteraciones estructurales, producto de una presión directa en la zona bilaminar por un desplazamiento hacia atrás del cóndilo de la mandíbula. Esta zona se relaciona con estructuras que juegan un rol importante en la fisiología del oído como el nervio auriculotemporal, el nervio de la cuerda del tímpano, así como la tuba auditiva y la musculatura del velo del paladar (Oyanguren et al., 2010).

Ligamento temporomandibular o Ligamento lateral. Hay una serie de ligamentos que cumplen la función de reforzar la unión de la cápsula articular (CA), según su disposición podemos diferenciar estos ligamentos en intracapsulares y extracapsulares, siendo necesario analizar si el ligamento temporomandibular o lateral forma parte de un ligamento capsular o extracapsular (Fuentes \& Ottone, 2017). Este posee dos porciones; una externa u oblicua y otra interna u horizontal. La porción externa está unida de forma anatómica y fisiológica a la Cápsula articular (CA) (Fig. 6), recubierta por células sinoviales y se comporta como un engrosamiento de ésta. Tiene forma de abanico, se inserta con su porción ancha desde la zona del arco cigomático hasta el tubérculo articular. Su porción inferior, en comparación con la superior es más estrecha, y se inserta en la zona postero-externa del cuello de la mandíbula (CCM). La porción interna, más laxa, se extendería hasta el polo lateral del cóndilo de la mandíbula abarcando de igual forma la ZP del DA. Su importancia radica en la limitación pasiva para restringir los movimientos mandibulares (Fuentes et al., 2016). Su función comienza ante los movimientos bordeantes o límite, a los que se oponen (Velayos, 2007). Este ligamento además logra proteger los tejidos retro discales y la excesiva distención del músculo pterigoideo lateral. Si bien, en condiciones normales permite un cierto grado de movimiento de la mandíbula hacia atrás desde la posición de intercuspídea, menor a $1 \mathrm{~mm}$ (Okesson, 2013); avanzar hacia la posición retrusiva mandibular no es una posición funcional, debido a que raramente se observa durante la masticación y deglución (Manns \& Díaz).

Músculo pterigoideo lateral, Cabeza superior. La posición articular funcional óptima es lograda a través de un correcto posicionamiento de los DA. En reposo, este concepto está influenciado por presiones interarticulares, la morfología de los propios discos y el tono de los músculos pterigoideos laterales superiores (Figs. 7 y 8). Estos últimos permiten que los DA roten de hacia adelante tanto como lo permitan los espacios discales, el grosor del margen posterior de los propios discos y ocasionalmente la lámina retrodiscal superior, que en reposo su retracción elástica es mínima o nula (Okesson, 2013). Entre sus funciones destaca jugar un papel fundamental en los movimientos mandibulares de cierre, retrusión y estabilización del cóndilo de la mandíbula y DA (Quirós et al., 2013).

La posible relación del musculo pterigoideo lateral con los Trastornos Temporomandibulares hace referencia a la hiper o hipo actividad del músculo, la leve coordinación entre las dos cabezas del músculo y/o una perturbación en el rol normal de la estabilización de la articulación temporomandibular. (Murray et al., 2004), ya que por ejemplo en un desplazamiento de disco sin reducción, la respuesta del músculo sería una contracción no fisiológica por una inadecuada interposición de los componentes articulares (Isberg). 

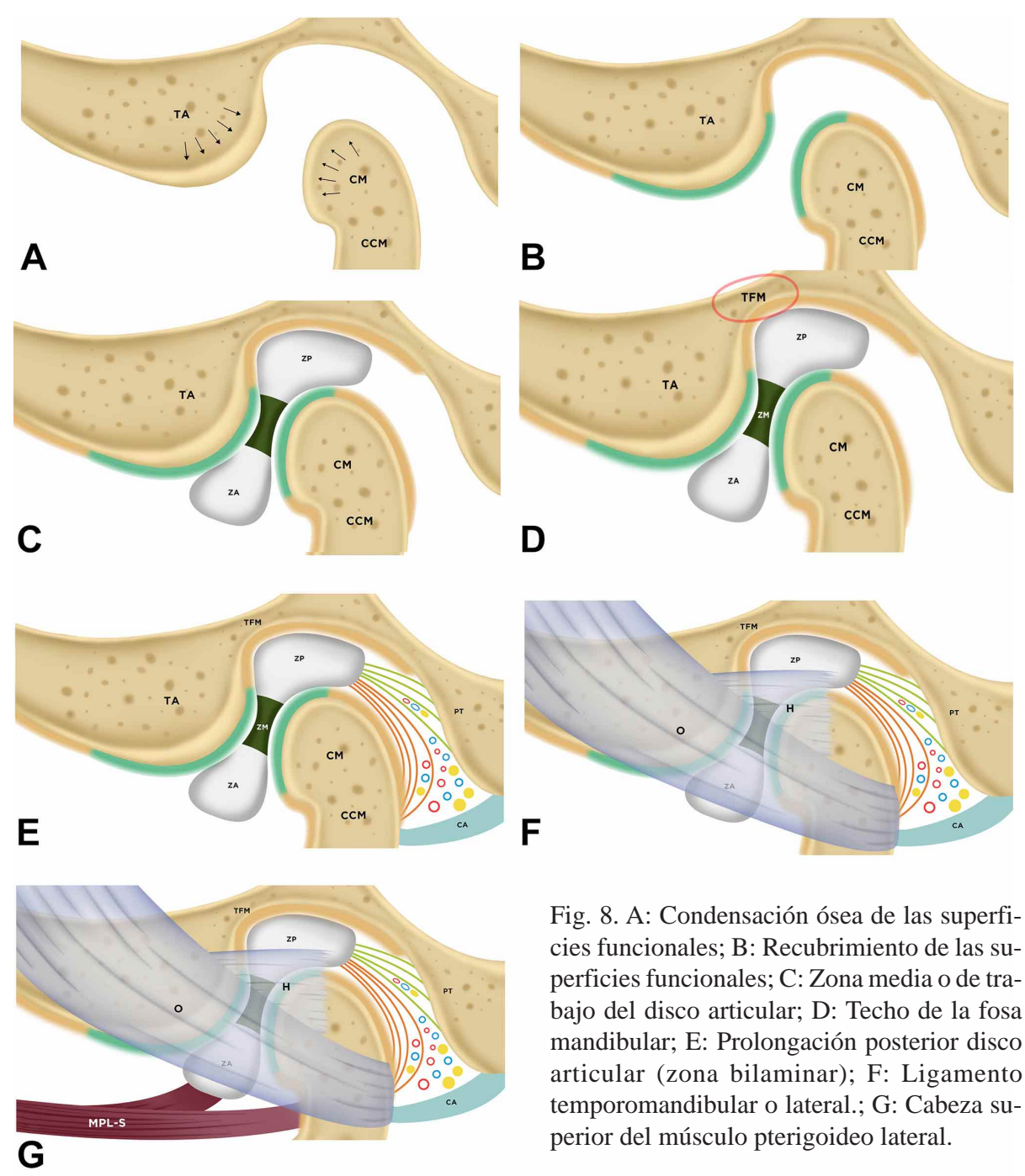

Fig. 8. A: Condensación ósea de las superficies funcionales; B: Recubrimiento de las superficies funcionales; $\mathrm{C}$ : Zona media o de trabajo del disco articular; D: Techo de la fosa mandibular; E: Prolongación posterior disco articular (zona bilaminar); F: Ligamento temporomandibular o lateral.; G: Cabeza superior del músculo pterigoideo lateral.

\section{CONCLUSIÓN}

Al ser la RC un concepto que, desde su definición, ha costado lograr el consenso de la comunidad científica, que ha ido mutando durante los años y que incluso en la actualidad no es comprendido en su totalidad por todos los estudiantes y profesionales del área de la odontología. Considerando su importancia en la planificación y éxito de diferentes tratamientos, se vuelve imprescindible lograr un conocimiento completo de las estructuras anatómicas involucradas en el proceso y las características propias de estas, en los odontólogos, ayudará a mejorar el desarrollo del ejercicio profesional, y la precisión con la que se reproduce o se genera una nueva RC.

Visto lo anterior es necesario tener en consideración las siguientes estructuras anatómicas: Condensación ósea de las superficies funcionales; Recubrimiento de las superficies funcionales; Zona media o de trabajo del disco articular; Techo de la fosa mandibular; Prolongación posterior disco articular (zona bilaminar); Ligamento temporomandibular o lateral. y Cabeza superior del músculo pterigoideo lateral. (Fig. 8). En conclusión siguiendo estas orientaciones anatomo-funcionales se complementa de buena forma con el concepto de RC.
FARFÁN, C.; QUIDEL, B. \& FUENTES, R. Anatomicalfunctional characteristics that guide the condylar position in mandibular fossa a centric relation. Narrative description. Int. J. Morphol., 38(5):1281-1287, 2020.

SUMMARY: The Central Relation (CR) is a concept that defines the ideal position of the mandibular condyle in the mandibular fossa. The position has been the subject of much discussion in the literature, noting changes as advancements in knowledge and technology are achieved. Thus, certain essential anatomical elements that are used as a guide for the clinician, are taken into account. The importance of being able to identify this position lies in the correct execution of both diagnostic and treatment procedures in various areas of dentistry. There are anatomical elements of the temporomandibular joint (TMJ) that improve understanding of the CR concept. These include: Bone condensation of the functional articular surfaces and their fibrocartilage coating; the middle portion of the joint disc, devoid of vessels and nerves. The roof of the mandibular fossa can become extremely thin, so it would not be considered an area to support load; the posterior prolongation of the articular disc (AD) Which is highly vascularized and innervated. The $\mathrm{AD}$ is also closely related to structures such as the temporomandibular or lateral ligament and the superior head of the lateral pterygoid muscle. Along with the previously described elements, this would allow proper functioning of the TMJ. The correct learning and use of these concepts contributes to a better exercise in the professional development of students and professionals in the area of dentistry.

KEY WORDS: Centric Relation; Functional anatomy; Temporomandibular joint; Oral physiology. 


\section{REFERENCIAS BIBLIOGRÁFICAS}

Aguila, F. \& Enlow, D. Crecimiento Craneofacial, Ortodoncia y Ortopedia. Actualidades Médico-Odontológicas Latinoamérica. Barcelona, Aguiram, 1993.

Caravadossi, A.; Guadalupe, M.; Odizzio, S.; Rué, G.; Vidal, A.; Villarnobo, F. \& Domínguez, D. Métodos de registro de la Relación Céntrica. ¿Son una necesidad en el diagnóstico y tratamiento de ortodoncia?. Actas Odontol., 9(1):59-64, 2012.

Dawson, P. E. Evaluation, Diagnosis and Treatment of Occlusal Problems. St. Louis, Mosby, 1974.

Dawson, P. E. New definition for relating occlusion to varying conditions of the temporomandibular joint. J. Prosthet. Dent., 74(6):619-27, 1995.

Dawson, P. E. Optimum TMJ condyle position in clinical practice. Int. J. Periodontics Restorative Dent., 5(3):10-31, 1985.

Estrella, G. Detección Precoz de los Desórdenes Temporomandibulares. Bogotá, AMOLCA, 2006.

Fuentes, R. \& Ottone, N. E. Proposal on inclusion and elimination of anatomical terms in Terminologia Anatomica corresponding to the temporomandibular joint. Int. J. Morphol., 35(1):12-5, 2017.

Fuentes, R.; Cantín, M.; Ottone, N. E. \& Bucchi, C. Characterization of bone components of the temporomandibular joint. A literature review. Int. J. Morphol., 33(4):1569-76, 2015.

Fuentes, R.; Ottone, N. E.; Bucchi, C. \& Cantín, M. Analysis of terms used in the literature to refer to temporomandibular joint capsule and joint ligaments. Int. J. Morphol., 34(1):342-50, 2016.

Glossary of Prosthodontic Terms. J. Prosthet. Dent., 58(6):713-62, 1987.

Gómez, M. E. \& Campos, A. Histología, Embriología, e Ingeniería Tisular Bucodental. $3^{\text {a }}$ ed. Buenos Aires, Médica Panamericana, 2009.

Isberg, A. Disfunción de la Articulación Temporomandibular. São Paulo, Artes Médicas Ltda., 2003.

Kandasamy, S.; Greene, C. S. \& Obrez, A. An evidence-based evaluation of the concept of centric relation in the 21st century. Quintessence Int., 49(9):755-60, 2018.

Langton, D. \& Eggleton, T. Functional Anatomy of the Temporomandibular Joint Complex. Tucson, INFORC Publications, 1992.

Lucia, V. O. Centric relation-Theory and practice. J. Prosthet. Dent., 10(5):849-56, 1960.

Manns, A. \& Biotti, J. Manual Práctico de Oclusión Dentaria. $2^{\mathrm{a}}$ ed. Caracas, AMOLCA, 2006.

Manns, A. \& Díaz, G. Sistema Estomatognático. Santiago de Chile, Empigraf, 1983.

Matamala, V. F.; Fuentes, F. R. \& Ceballos, C. M. Morphology and morphometry of the temporomandibular joint disc in human fetus and adults. Int. J. Morphol., 24(2):245-50, 2006.

McCollum, B. B. Considering the mouth as a functioning unit as a basis of a dental diagnosis. J. S. Calif. Dent. Assoc., 5:268-76, 1938.

Murray, G. M.; Phanachet, I.; Uchida, S. \& Whittle, T. The human lateral pterygoid muscle: a review of some experimental aspects and possible clinical relevance. Aust. Dent. J., 49(1):2-8, 2004.

Okesson, J. Tratamiento de Oclusión y Afecciones Temporomandibulares. $5^{\text {a }}$ ed. Madrid, Mosby, 2003.

Okesson, P. Tratamiento de Oclusión y Afecciones Temporomandibulares. $7^{\mathrm{a}}$ ed. Barcelona, Mosby, 2013.

Orozco Varo, A.; Arroyo Cruz, G.; Martinez de Fuentes, R.; Ventura de la Torre, J.; Cañadas Rodríguez, D. \& Jiménez Castellanos, E. Relación céntrica: revisión de conceptos y técnicas para su registro. Parte I. $A v$. Odontoestomatol., 24(6):365-8, 2008.

Orozco Varo, A.; Arroyo Cruz, G.; Martinez de Fuentes, R.; Ventura de la Torre, J.; Cañadas Rodríguez, D. \& Jiménez Castellanos, E. Relación céntrica: revisión de conceptos y técnicas para su registro. Parte II. $A v$. Odontoestomatol., 24(6):369-76, 2008.

Oyanguren, F. R.; Wurgaft, D. R. \& Montenegro, R. M. A. Histological and histochemical evaluation of collagen and elastic fibers in human TMJ tissue. Int. J. Odontostomat., 4(3):277-84, 2010.
Quirós, J. G.; Pérez Osorio, L. J. \& Calderón, J. C. Influencia del músculo pterigoideo lateral en el crecimiento del cartílago condilar mandibular. Rev. Cienc. Salud, 11(1):105-19, 2013.

Ramfjord, A. \& Ash, M. Oclusión. 2 2a ed. Filadelfia, Interamericana, 1972.

Rinchuse, D. J. \& Kandasamy, S. Centric relation: A historical and contemporary orthodontic perspective. J. Am. Dent. Assoc., 137(4):494501, 2006.

Rubiano, C. M. Placa Neuromiorelajante: Elaboración y Mantenimiento Paso a Paso. Bogotá, Actualidades Médico-Odontológicas Latinoamérica, 1993.

Stankovic, S.; Vlajkovic, S.; Boskovic, M.; Radenkovic, G.; Antic, V. \& Jevremovic, D. Morphological and biomechanical features of the temporomandibular joint disc: an overview of recent findings. Arch. Oral Biol., 58(10):1475-82, 2013.

Stocum, D. L. \& Roberts, W. E. Part I: Development and physiology of the temporomandibular joint. Curr. Osteoporos. Rep., 16(4):360-8, 2018.

The Glossary of Prosthodontic Terms: Ninth Edition. J. Prosthet. Dent., 117(5S):e1-e105, 2017.

Velayos, J. L. \& Santana, H. D. Anatomía de la Cabeza con Enfoque Odontoestomatológico. $3^{\mathrm{a}}$ ed. Madrid, Médica Panamericana, 2001.

Velayos, J. L. Anatomía de la Cabeza. $4^{\mathrm{a}}$ ed. Madrid, Médica Panamericana, 2007.

\section{Corresponding author:}

Prof. Dr. Ramón Fuentes Fernández

Research Centre for Dental Sciences

Dental School

Universidad de La Frontera

Av. Francisco Salazar 1145

Temuco

CHILE

Email: ramon.fuentes@ufrontera.cl

Recibido : 29-02-2020

Aceptado: 29-04-2020 